\title{
Contribution of Serum Biomarkers to Prognostic Assessment in Patients With Oligometastatic Prostate Cancer
}

\author{
CARSTEN NIEDER $^{1,2}$, ASTRID DALHAUG $^{1}$ and ADAM PAWINSKI ${ }^{1}$ \\ ${ }^{1}$ Department of Oncology and Palliative Medicine, Nordland Hospital, Bod $\phi$, Norway; \\ ${ }^{2}$ Department of Clinical Medicine, Faculty of Health Sciences, \\ UiT - The Arctic University of Norway, Troms $\phi$, Norway
}

\begin{abstract}
Background/Aim: The aim of this study was to analyze the prognostic impact of biomarkers, such as serum lactate dehydrogenase $(\mathrm{LDH})$, in patients with oligometastatic castration-resistant prostate cancer, arbitrarily defined as a maximum of five metastatic lesions. Patients and Methods: This was a retrospective single-institution analysis. Overall 34 patients were included, all of whom received first-line docetaxel without ablative local treatment. Results: Twelve patients (35\%) had elevated LDH $(\geq 255 \mathrm{U} / \mathrm{l})$. Their median survival was significantly shorter than that of patients with normal LDH. Due to an interaction with other biomarkers, multivariate Cox regression analysis was performed. The latter showed that serum hemoglobin was the only significant predictor of survival. Conclusion: Correct diagnosis of oligometastatic disease is not trivial, because all radiological modalities are limited by certain thresholds for detection of small metastases. Serum biomarkers may reflect the total burden of malignant disease. However, this relatively small study did not clearly demonstrate that elevation of LDH may be useful for clinical decision-making, e.g. in terms of adding local treatment for all sites of metastatic spread.
\end{abstract}

Increasing attention has recently been paid to patients with oligometastatic disease, i.e. a state between curable locoregional disease and incurable dissemination throughout large parts of the body (1-4). If only few distant metastases are present, ablative local treatment or resection of all these lesions might result in longer survival than historically

This article is freely accessible online.

Correspondence to: Dr. Carsten Nieder, Department of Oncology and Palliative Medicine, Nordland Hospital, 8092 Bodø, Norway. Tel: +47 75578449, Fax: +47 75534975, e-mail: carsten.nieder@nlsh.no

Key Words: Palliative chemotherapy, docetaxel, prostate cancer, oligometastases, prognostic factors, biomarkers, lactate dehydrogenase. observed (5-7). However, not all patients become long-term survivors, a finding that emphasizes the challenges associated with detecting true oligometastatic disease. If additional micrometastases go undetected, rapid disease progression might be encountered. It would, therefore, be prudent to include staging examinations that are not limited by the detection thresholds of radiological methods (8-10). For example, the number of circulating tumor cells, cell-free DNA or other emerging biomarkers may aid in the detection of true and pseudo-oligometastatic disease $(11,12)$. Given that many patients, especially in low- and middle-income countries, lack access to advanced imaging and biomarker evaluations, widely available and inexpensive biomarkers may also contribute to improved prognostic assessment and decision-making. Therefore, we studied the prognostic impact of serum lactate dehydrogenase (LDH), prostatespecific antigen (PSA), alkaline phosphatase (ALP) and hemoglobin in patients with oligometastatic castrationresistant prostate cancer (MCRPC) who received first-line chemotherapy with docetaxel. We hypothesized that such biomarkers might reflect the overall disease burden in patients presumed to harbor oligometastases. If true, patients with elevated serum biomarkers due to otherwise undetectable additional metastases are expected to have poorer survival and may be spared the burden of aggressive local treatment.

\section{Patients and Methods}

Patients and treatment. A retrospective study of all patients with MCRPC and a maximum of five distant metastases treated with first-line docetaxel at our hospital was performed. An existing database was used to identify eligible patients (13). These patients had not received docetaxel for hormone-sensitive metastatic disease earlier. Further treatment was individualized and consisted of additional lines of systemic therapy (Ra-223, enzalutamide, abiraterone acetate, cabazitaxel) or best supportive care. Systemic treatment was usually prescribed as judged appropriate by the patients' clinical oncologists and was tailored to performance status (typically $0-1$ ), organ function and comorbidity. The patients 
Table I. Patient characteristics.

\begin{tabular}{lc}
\hline Baseline parameter & Value \\
\hline Bone metastases only, n (\%) & $16(47)$ \\
Lymph node metastases only, n (\%) & $9(26)$ \\
Visceral metastases only, n (\%) & $2(6)$ \\
Bone and lymph node metastases, n (\%) & $7(21)$ \\
Median serum PSA (range), $\mu \mathrm{g} / 1$ & $93(6-1487)$ \\
Median serum ALP (range), U/1 & $123(47-916)$ \\
Median serum LDH (range), U/1 & $211(146-675)$ \\
Median hemoglobin (range), g/dl & $13.6(9.6-16.0)$ \\
\hline
\end{tabular}

ALP: Alkaline phosphatase; LDH: lactate dehydrogenase; PSA prostate-specific antigen.

were treated between January 01, 2007 and December 31, 2017. Staging consisted of computed tomography and bone isotope scan. Biomarkers were assessed at the start of chemotherapy or within the preceding week (the normal value was defined as $<255 \mathrm{U} / 1$ for $\mathrm{LDH},<105 \mathrm{U} / 1$ for ALP, and 13.4-17.0 g/dl for hemoglobin).

Statistical methods. Overall survival (time to death) from the first day of chemotherapy was calculated employing the Kaplan-Meier method, and different groups were compared using the log-rank test (SPSS 25; IBM Corp., Armonk, NY, USA). Only four patients were censored after a median follow-up of 48 months. Date of death was known for all other patients. A Cox forward conditional regression model was employed for multivariate analysis. Statistical significance was defined as $p<0.05$ throughout this study.

\section{Results}

Patient characteristics. A total of 34 patients were identified from the database. Their median age was 69 (range=56-78) years. Six patients $(18 \%)$ already had distant metastases when diagnosed with prostate cancer, the others developed metachronous metastases. Nine patients $(26 \%)$ had pelvic lymph node metastases $(\mathrm{N}+$ disease) at first diagnosis. Thirteen patients $(38 \%)$ were initially treated with curative radiotherapy or radical prostatectomy. Two patients $(6 \%)$ received palliative radiotherapy to the prostate before chemotherapy. Further patient characteristics are shown in Table I.

Treatment for MCRPC. Twenty patients (59\%) received docetaxel every 3 weeks $\left(75 \mathrm{mg} / \mathrm{m}^{2}\right)$ and the remainder every 2 weeks or every week at lower doses. Oligometastases were not treated with local measures such as surgery or ablative radiotherapy. Bisphosphonates were given to 11 patients (32\%) concomitant to chemotherapy. Except for nine patients $(26 \%)$, further systemic treatment with approved agents (Ra-223, enzalutamide, abiraterone acetate, cabazitaxel) was given after disease progression on docetaxel (14).
Survival. The median overall survival of the whole patient cohort was 27.7 months. As shown in Figure 1, LDH was significantly associated with survival. Survival beyond 5 years was observed in patients with normal LDH only. Survival was not significantly associated with previous treatment of the primary tumor (surgery or curative radiotherapy vs. none), bisphosphonate use, patterns of metastasis (bone only, lymph nodes only etc.), age, ALP and PSA. However, patients with a normal hemoglobin level survived significantly longer than their counterparts with abnormally low values. In the Cox regression model, hemoglobin retained statistical significance $(p=0.008)$, whereas LDH did not $(p=0.21)$.

\section{Discussion}

We performed a retrospective study on patients with a limited number of distant metastases (maximum five in total) and evaluated biomarkers which previously have been studied in different other settings $(15,16)$. We found that elevated LDH and reduced hemoglobin were associated with significantly shorter survival, however in multivariate analysis, hemoglobin outperformed LDH.

Limitations of the present study include the limited number of patients, statistical power of subgroup analyses, and retrospective design. The presence of oligometastatic disease was not confirmed by positron-emission tomography (PET) staging. However, PET staging is also associated with false-negative and otherwise inaccurate findings (7). Moreover, the availability of PET is limited or, in some low- and middle-income countries, even non-existent. Under these circumstances, readily available biomarkers might complement computed tomographic- and isotope scan-based staging, with the ultimate goal of avoiding overtreatment in patients with pseudo-oligometastatic disease. Emerging biomarkers such as those based on circulating tumor cells are not widely available yet (17), in contrast to hemoglobin, LDH or ALP measurement.

We limited this analysis to patients treated with first-line docetaxel rather than a variety of different approved options in order to eliminate bias arising from differences in first-line systemic treatment. As recently discussed by different groups, aggressive local treatment of oligometastatic cancer is expected to improve survival $(2-4,18)$. Even in the absence of metastasis-directed local treatment, we found long-term survivors in the group with normal hemoglobin (and also LDH). Our results suggest that readily available blood tests may add value when designing further clinical trials and in resource-limited settings.

\section{Conclusion}

Correct diagnosis of oligometastatic disease is not trivial because all radiological modalities are limited by certain thresholds for the detection of small metastases. Serum 


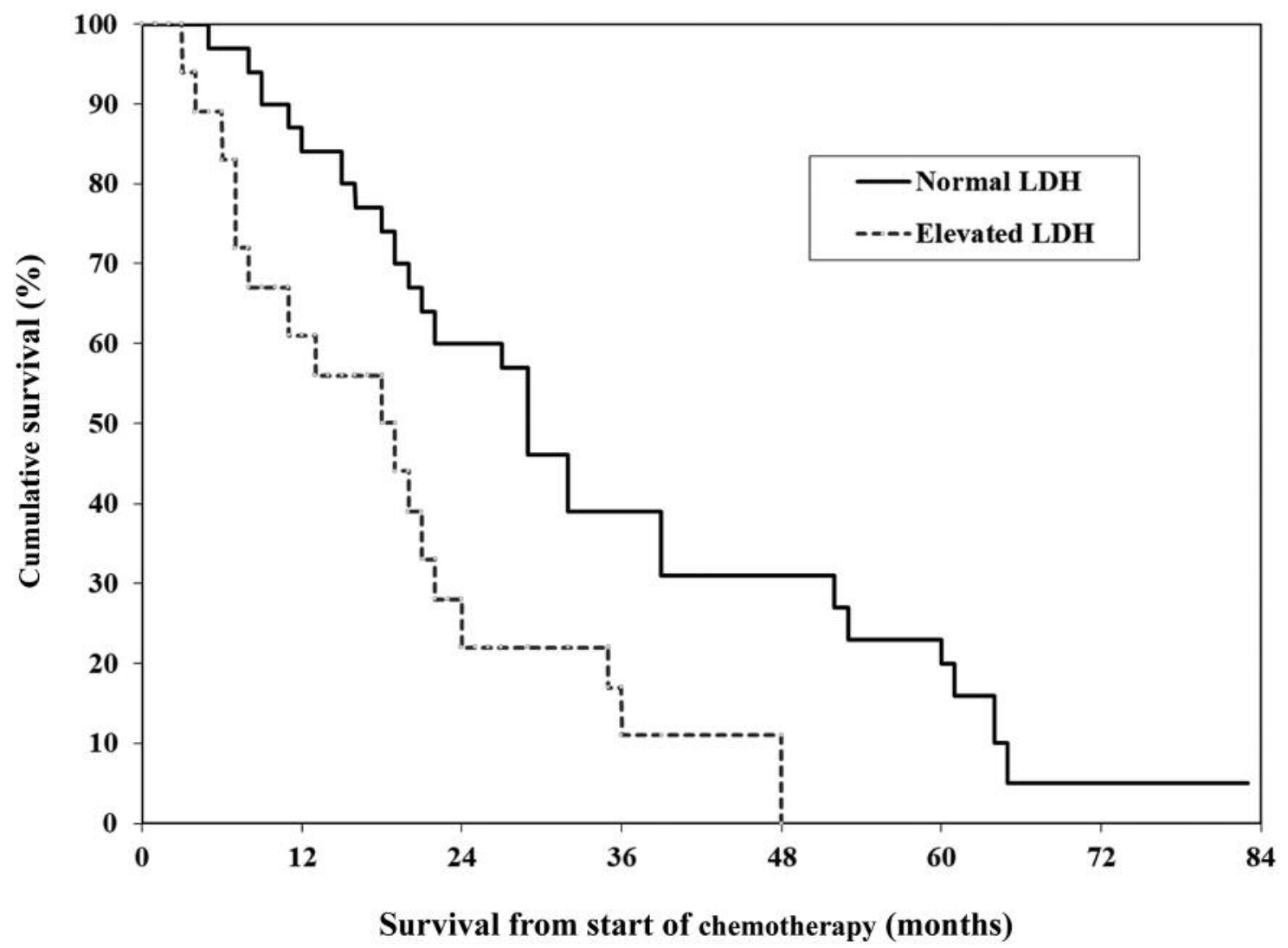

Figure 1. Actuarial overall survival stratified by serum lactate dehydrogenase (LDH). Normal LDH: Median 29.8 months, elevated LDH: median 20.0 months $(p=0.036)$.

biomarkers may reflect the total burden of malignant disease. However, this relatively small study did not clearly demonstrate that elevation of LDH may be useful for clinical decision-making, e.g. in terms of adding local treatment for all sites of metastatic spread.

\section{Conflicts of Interest}

The Authors declare that they have no competing interests.

\section{References}

1 Tosoian JJ, Gorin MA, Ross AE, Pienta KJ, Tran PT and Schaeffer EM: Oligometastatic prostate cancer: Definitions, clinical outcomes, and treatment considerations. Nat Rev Urol 14: 15-25, 2017.

2 Oertel M, Scobioala S, Kroeger K, Baehr A, Stegger L, Haverkamp U, Schäfers $M$ and Eich HT: Worth a local treatment? - Analysis of modern radiotherapy concepts for oligometastatic prostate cancer. Radiat Oncol 13: 185, 2018.

3 Siva S, Bressel M, Murphy DG, Shaw M, Chander S, Violet J, Tai KH, Udovicich C, Lim A, Selbie L, Hofman MS, Kron T, Moon D, Goad J, Lawrentschuk N and Foroudi F: Stereotactic ablative body radiotherapy (SABR) for oligometastatic prostate cancer: A prospective clinical trial. Eur Urol 74: 455-462, 2018.

4 Palma DA, Louie AV and Rodrigues GB: New strategies in stereotactic radiotherapy for oligometastases. Clin Cancer Res 21: 5198-5204, 2015.

5 Pasqualetti F, Panichi M, Sainato A, Baldaccini D, Cocuzza P, Gonnelli A, Montrone S, Molinari A, Barbiero S, Bruschi A, Notini E, Ursino S, Mazzotti V, Morganti R, Coraggio G, Cantarella M, Erba PA and Paiar F: Image-guided stereotactic body radiotherapy in metastatic prostate cancer. Anticancer Res 38: 3119-3122, 2018.

6 Franzese C, Zucali PA, Di Brina L, D'Agostino G, Navarria P, Franceschini D, Santoro A and Scorsetti M: The efficacy of stereotactic body radiation therapy and the impact of systemic treatments in oligometastatic patients from prostate cancer. Cancer Med 7: 4379-4386, 2018.

7 Lecouvet FE, Oprea-Lager DE, Liu Y, Ost P, Bidaut L, Collette L, Deroose CM, Goffin K, Herrmann K, Hoekstra OS, Kramer G, Lievens Y, Lopci E, Pasquier D, Petersen LJ, Talbot JN, Zacho H, Tombal B and deSouza NM: Use of modern imaging methods to facilitate trials of metastasis-directed therapy for oligometastatic disease in prostate cancer: A consensus recommendation from the EORTC Imaging Group. Lancet Oncol 19: e534-e545, 2018. 
8 deSouza NM, Liu Y, Chiti A, Oprea-Lager D, Gebhart G, Van Beers BE, Herrmann $\mathrm{K}$ and Lecouvet FE: Strategies and technical challenges for imaging oligometastatic disease: Recommendations from the European Organisation for Research and Treatment of Cancer imaging group. Eur J Cancer 91: 153163,2018

9 Cysouw M, Bouman-Wammes E, Hoekstra O, van den Eertwegh A, Piet M, van Moorselaar J, Boellaard R, Dahele M and OpreaLager D: Prognostic value of [(18)F]-fluoromethylcholine positron-emission tomography/computed tomography before stereotactic body radiation therapy for oligometastatic prostate cancer. Int J Radiat Oncol Biol Phys 101: 406-410, 2018.

10 Evangelista L, Cuppari L, Guttilla A, Gardi M, Agostini A, Ruggera L, Basso U and Saladini G: Oligometastatic recurrent prostate cancer detects by fluorine-18-choline positron-emission tomography/computed tomography in patients with prostatespecific antigen levels of up to $5 \mathrm{ng} / \mathrm{ml}$. Nucl Med Commun 39: 260-267, 2018.

11 Di Nunno V, Gatto L, Santoni M, Cimadamore A, Lopez-Beltran A, Cheng L, Scarpelli M, Montironi R and Massari F: Recent advances in liquid biopsy in patients with castration-resistant prostate cancer. Front Oncol 8: 397, 2018.

12 Zainfeld D and Goldkorn A: Liquid biopsy in prostate cancer: Circulating tumor cells and beyond. Cancer Treat Res 175: 87104,2018

13 Nieder C, Haukland E, Pawinski A and Norum J: Seven-month prostate-specific antigen (PSA) is prognostic in patients with prostate cancer initially diagnosed with distant metastases. Med Oncol 35: 46, 2018.
14 Norum J and Nieder C: Treatments for metastatic prostate cancer (mPC): A review of costing evidence. Pharmacoeconomics 35: 1223-1236, 2017.

15 Sartor O, Coleman RE, Nilsson S, Heinrich D, Helle SI, O'Sullivan JM, Vogelzang NJ, Bruland Ø, Kobina S, Wilhelm S, $\mathrm{Xu}$ L, Shan M, Kattan MW and Parker C: An exploratory analysis of alkaline phosphatase, lactate dehydrogenase, and prostate-specific antigen dynamics in the phase 3 ALSYMPCA trial with radium-223. Ann Oncol 28: 1090-1097, 2017.

16 Chi KN, Kheoh T, Ryan CJ, Molina A, Bellmunt J, Vogelzang NJ, Rathkopf DE, Fizazi K, Kantoff PW, Li J, Azad AA, Eigl BJ, Heng DY, Joshua AM, de Bono JS and Scher HI: A prognostic index model for predicting overall survival in patients with metastatic castration-resistant prostate cancer treated with abiraterone acetate after docetaxel. Ann Oncol 27: 454-460, 2016.

17 Heller G, Fizazi K, McCormack R, Molina A, MacLean D, Webb IJ, Saad F, de Bono JS and Scher HI: The added value of circulating tumor cell enumeration to standard markers in assessing prognosis in a metastatic castration-resistant prostate cancer population. Clin Cancer Res 23: 1967-1973, 2017.

18 Stenman M, Sinclair G, Paavola P, Wersäll P, Harmenberg U and Lindskog M: Overall survival after stereotactic radiotherapy or surgical metastasectomy in oligometastatic renal cell carcinoma patients treated at two Swedish centres 2005-2014. Radiother Oncol 127: 501-506, 2018.

Received November 2, 2018

Revised November 22, 2018 Accepted November 28, 2018 